\title{
Rehabilitation and cross-language transfer in bilingual aphasia: towards a computational model
}

\author{
Uli Grasemann 1*, Chaleece Sandberg ${ }^{2}$, Swathi Kiran², Risto Miikkulainen ${ }^{1}$ \\ From Nineteenth Annual Computational Neuroscience Meeting: CNS*2010 \\ San Antonio, TX, USA. 24-30 July 2010
}

Bilingual aphasia, defined as a loss of one or both languages in bilingual individuals that results from left hemisphere damage, is of increasing interest worldwide because more than half of the world's population is bilingual. Despite the importance of this topic in an increasingly bilingual world, disparities in diagnosis and treatment of bilingual aphasia continue to exist. Current research on this topic lacks specific recommendations on which languages should be trained in a bilingual aphasic individual and to what extent cross-language transfer occurs subsequent to rehabilitation. Factors contributing to the paucity of research in this area relate to the multitude of possible language combinations in a bilingual individual, the relative competency of the two languages of the bilingual individual, and the effect of focal brain damage on bilingual language representation. It is, however, unfeasible to examine these issues clinically without undertaking a large scale longitudinal study in this population.

As a potential solution to this problem, we are currently developing a computational model that will make a systematic examination of cross-language transfer subsequent to rehabilitation possible. The model is an extended version of DISLEX, a simulation of lexical access in humans based on self-organizing maps. For the present work, the model was extended to simulate a bilingual language system in which language representations can vary by age of acquisition and relative proficiency. The model can be lesioned systematically in different ways to simulate the damage leading to bilingual aphasia. Subsequent training using only one of the model's two languages will then be performed to simulate treatment in aphasic individuals, and will make it possible to examine how age of acquisition, level of pre- morbid language proficiency, and post-morbid language performance influence the nature and degree of crosslanguage transfer.

Here we report on the progress of the project so far. An outline of the model is given, including its implementation as well as its theoretical underpinnings in light of recent empirical evidence. The methods and input data used to train the model and to simulate treatment and clinical tests are described. First findings are presented that demonstrate that the model is able to match the available data on individual patients before the onset of aphasia. Starting with such models of individual patients, possible effects of lesions to the model and their ability to simulate post-morbid language performance are then discussed. Finally, we discuss how the model could in the future complement clinical research in determining the best approach to treating aphasia in bilingual patients, possibly in a way that is tailored to individual patients.

\section{Author details}

${ }^{1}$ Department of Computer Science, The University of Texas at Austin, Austin, TX 78712, USA. ²Department of Speech, Language \& Hearing Sciences, Boston University, Boston, MA 02215, USA.

Published: 20 July 2010

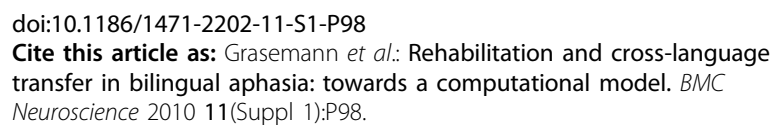

* Correspondence: uli@cs.utexas.edu

'Department of Computer Science, The University of Texas at Austin, Austin, TX 78712, USA 